\title{
La Venta de Borondo: Patrimonio cultural manchego ligado al Quijote en peligro
}

\section{David Cejudo Loro a, Julio Orellana López de la Franca ${ }^{a}$ y Miguel Torres Más ${ }^{a}$}

${ }^{a}$ Asociación Cultural Venta de Borondo y Patrimonio Manchego, Calle Francisco Pérez s/n, 13250 Daimiel (Ciudad Real). acventaborondo@gmail.com

\begin{abstract}
Resumen
La Venta de Borondo es una venta manchega del siglo XVI situada en Daimiel (Ciudad Real) que se encuentra en un grave estado de deterioro. Esta hospedería se construyó en un enclave importante junto al Camino Real que conectaba Almagro con el Levante y sureste español. La venta fue declarada en 2007 Bien de Interés Cultural en la categoría de Monumento; sin embargo, el edificio, de propiedad privada, no fue objeto de restauración llegando su situación a ser crítica desde 2016. La grave situación del monumento movilizó a la sociedad daimieleña, traduciéndose en la fundación de la Asociación Cultural Venta de Borondo y Patrimonio Manchego, quien en 2018 ejecutó una intervención de urgencia para la restauración del torreón suroeste de la venta.
\end{abstract}

Palabras clave: venta, camino real, restauración, arquitectura tradicional, asociacionismo, La Mancha, Cervantes.

\begin{abstract}
Borondo's Inn is a Manchego inn from the 16th century located in Daimiel (Ciudad Real), which is in a serious state of deterioration. This inn was built in an important region beside Real Road that connected Almagro with the Levante and southeast of Spain. The inn was declared in 2007 as a Site of Cultural Interest in the Monument category. However, the building, which is a private property, was not restored, and its situation became critical since 2016. The severe situation of the monument encouraged the society from Daimiel, resulting in the founding of the "Venta de Borondo y Patrimonio Manchego Association", who carried out an emergency intervention in 2018 for the restoration of the inn's southeast tower.
\end{abstract}

Keywords: inn, royal road, heritage restoration, traditional architecture, associationism, La Mancha, Cervantes. 


\section{La Venta de Borondo: Aproximación histórico-artística}

La Venta de Borondo es una hospedería manchega del siglo XVI situada en la localidad de Daimiel (Ciudad Real). En 2007 fue declarada Bien de Interés Cultural en la categoría de Monumento por su valor histórico, artístico y literario, al ser una tipología arquitectónica vinculada a la genial obra de Don Miguel de Cervantes (JCCM, 2007, p. 16.436). En estas ventas se desarrollan varios capítulos de la novela, siendo la Venta de Borondo de gran importancia, ya que es una de las últimas ventas coetáneas al autor, apareciendo ya referenciada en 1575 en las Relaciones Topográficas de Felipe II (Campos y Fernández de Sevilla, 2009, p. 419).

El objetivo de esta publicación es explicar el conjunto de factores históricos, arquitectónicos y de acción social que ponen de manifiesto el valor cultural de la Venta de Borondo. Para su elaboración se ha recurrido a las distintas fuentes bibliográficas existentes y se ha profundizado en el trabajo que ha venido realizando la Asociación Cultural Venta de Borondo y Patrimonio Manchego (en adelante AC Venta de Borondo) desde el año 2016.

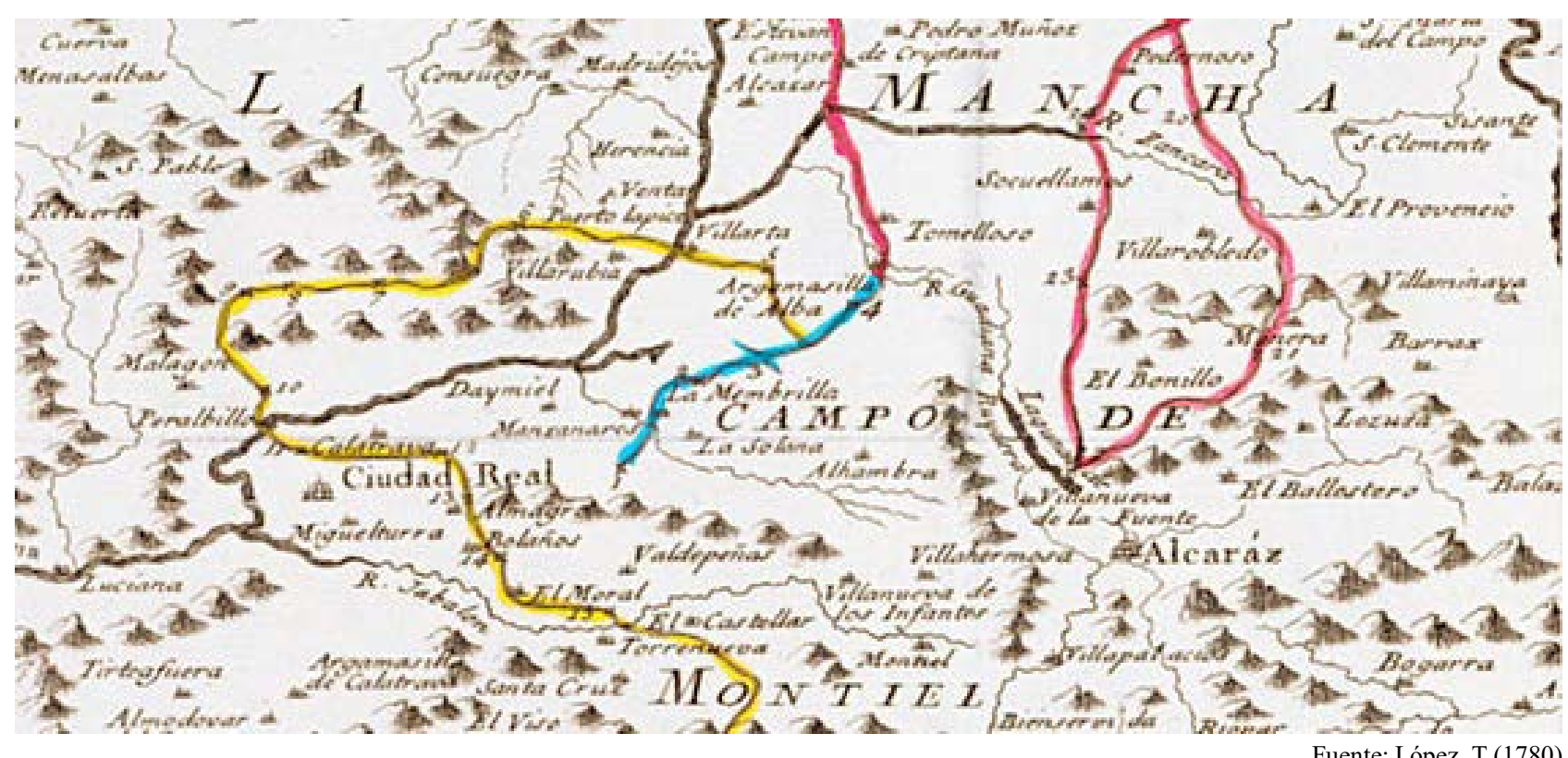

Fig. 1 Mapa ruta del Quijote de Tomás López, 1780

En relación a su vinculación con el patrimonio cultural ligado a El Quijote se pueden referenciar una serie de factores que ponen de manifiesto su relevancia en este ámbito. Diversos autores han intentado identificar las localizaciones donde se desarrollaron las hazañas de El hingenioso hidalgo Don Quijote de la Mancha. El geógrafo Tomás López será el primero en trazar un mapa de la ruta del Quijote, localizando la venta donde fue armado caballero en un punto intermedio entre Almagro y Manzanares, no pudiendo ser otra que la Venta de Borondo (Fig. 1). Esta propuesta será criticada en 1790 en un folleto anónimo que propone como alternativa la Venta Quesada por encontrarse junto al Camino Real que conectaba Madrid con Sevilla (Anónimo, 1790, pp. 29-30). A pesar de todo, la identificación de este edificio como "venta del Quijote" llegará hasta el siglo XX. Ángel Dotor firmará un artículo en la revista Blanco y Negro donde describe ésta como la venta «...en la que Don Quijote fue armado caballero» (Dotor, 1925, p. 49).

A nivel arquitectónico en la venta se pueden distinguir dos partes bien diferenciadas: un edificio principal de dos alturas y una serie de edificios anexos. El edificio principal sería la hospedería original construida siguiendo las técnicas de la arquitectura tradicional en tierra. Sus muros se levantan con la técnica del tapial calicostrado, su cubierta se recubre con teja curva árabe y las estancias se distribuyen en torno a un patio empedrado donde se sitúa un pozo. En su esquina suroeste se eleva un torreón, originariamente con cuatro grandes vanos que en época contemporánea serían cegados para crear un palomar (Fig. 2). Su acceso principal se sitúa en el alzado este, la puerta está flanqueada por un pórtico de sillería decorado con dos ménsulas de querubines, un escudo de armas sobre el dintel y dos medias columnas con capiteles decorados con angelotes. Desgraciadamente la media columna derecha y los querubines fueron objeto de expolio (Cejudo, 2013, pp. 71-74). 


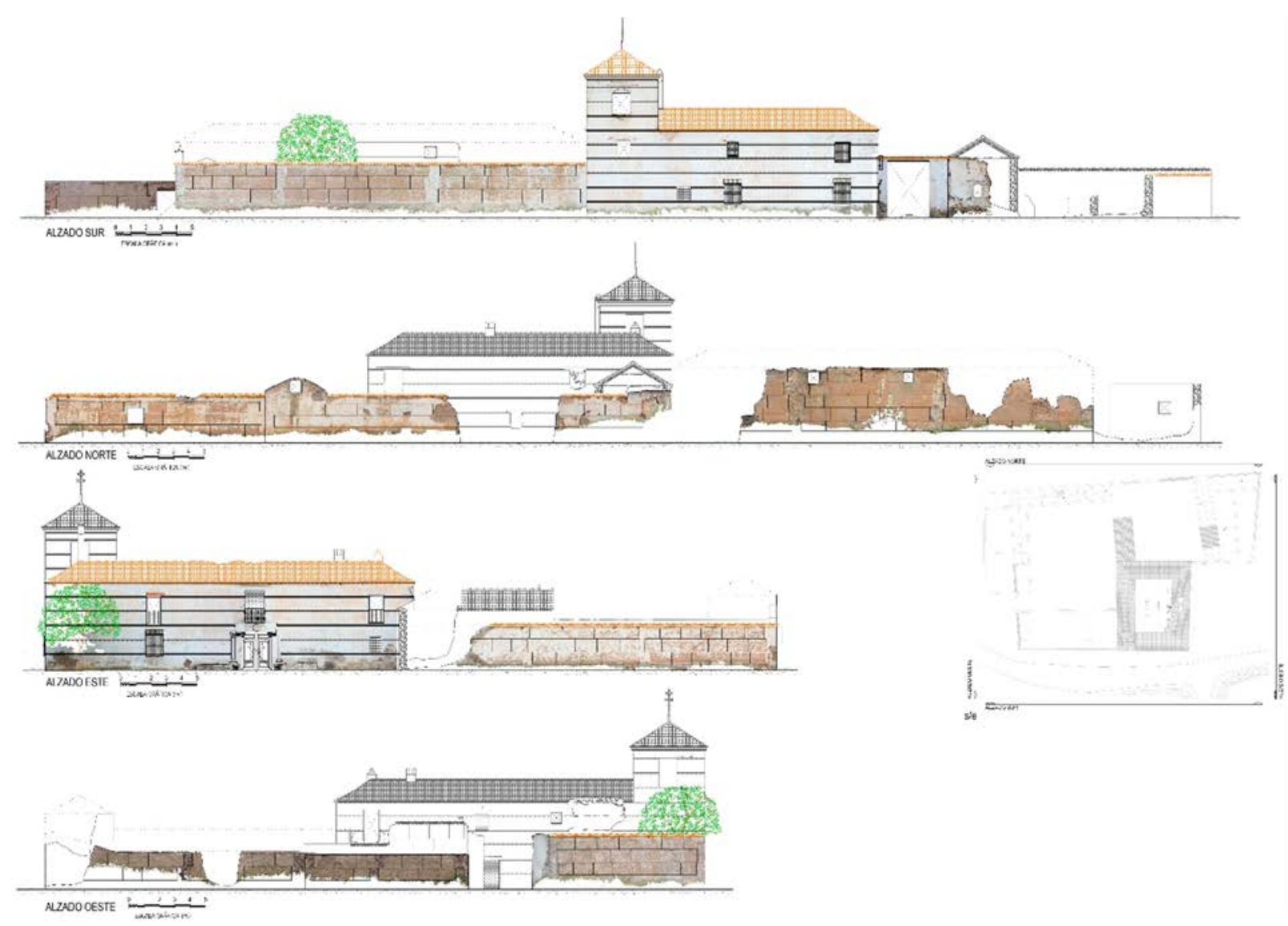

[Figura de David Cejudo]. (Alzados Venta de Borondo. 2012) (Cejudo, 2012)

Fig. 2 Venta de Borondo. Conjunto de alzados exteriores

Los edificios anexos serán construidos entre los siglos XIX y XX cuando la Venta de Borondo se transforme en una explotación agropecuaria. Se levantarán cocinas y establos en torno a dos grandes patios (Fig. 3) con diversa funcionalidad, siendo utilizado por la trashumancia hasta bien entrado el siglo XX. La calidad del muro es inferior al carecer de calicostrado permitiendo que su deterioro haya sido más rápido y que se encuentre actualmente en estado de ruina.

Este monumento es actualmente de propiedad privada, se encuentra en estado de abandono y está en peligro de ruina pese a su catalogación como Bien de Interés Cultural. La aparición de graves patologías en 2013 justificó su inclusión en la Lista Roja del Patrimonio de Hispania Nostra, pero no será hasta 2016 cuando la situación alcance tal gravedad que se traducirá en la fundación de una asociación en defensa del patrimonio cultural manchego.

\section{La Asociación Cultural Venta de Borondo y Patrimonio Manchego}

En 2016 la situación de la Venta de Borondo era crítica. El torreón suroeste había perdido una sección importante del muro de tapial, que junto a la pérdida de la cornisa y de parte del muro del alzado oeste contiguo, hacía previsible su derrumbe inminente. Pronto saltó la alarma entre varios vecinos de Daimiel y se organizó una jornada reivindicativa pidiendo a las administraciones una intervención de urgencia. Pero ante la falta de respuesta política, un grupo de ciudadanos de diversos sectores profesionales decidió fundar la AC Venta de Borondo con la intención de impulsar esa intervención.

El primer paso de la asociación fue reunirse con los propietarios de la venta, consiguiéndose el compromiso de ceder o donar la Venta de Borondo a cualquier administración que se comprometiera a restaurarla. El siguiente objetivo marcado por la asociación consistió en el desarrollo de un proyecto global de restauración por fases y una propuesta de puesta en valor del monumento, que se presentaría ante diferentes administraciones públicas y empresas de la localidad. 


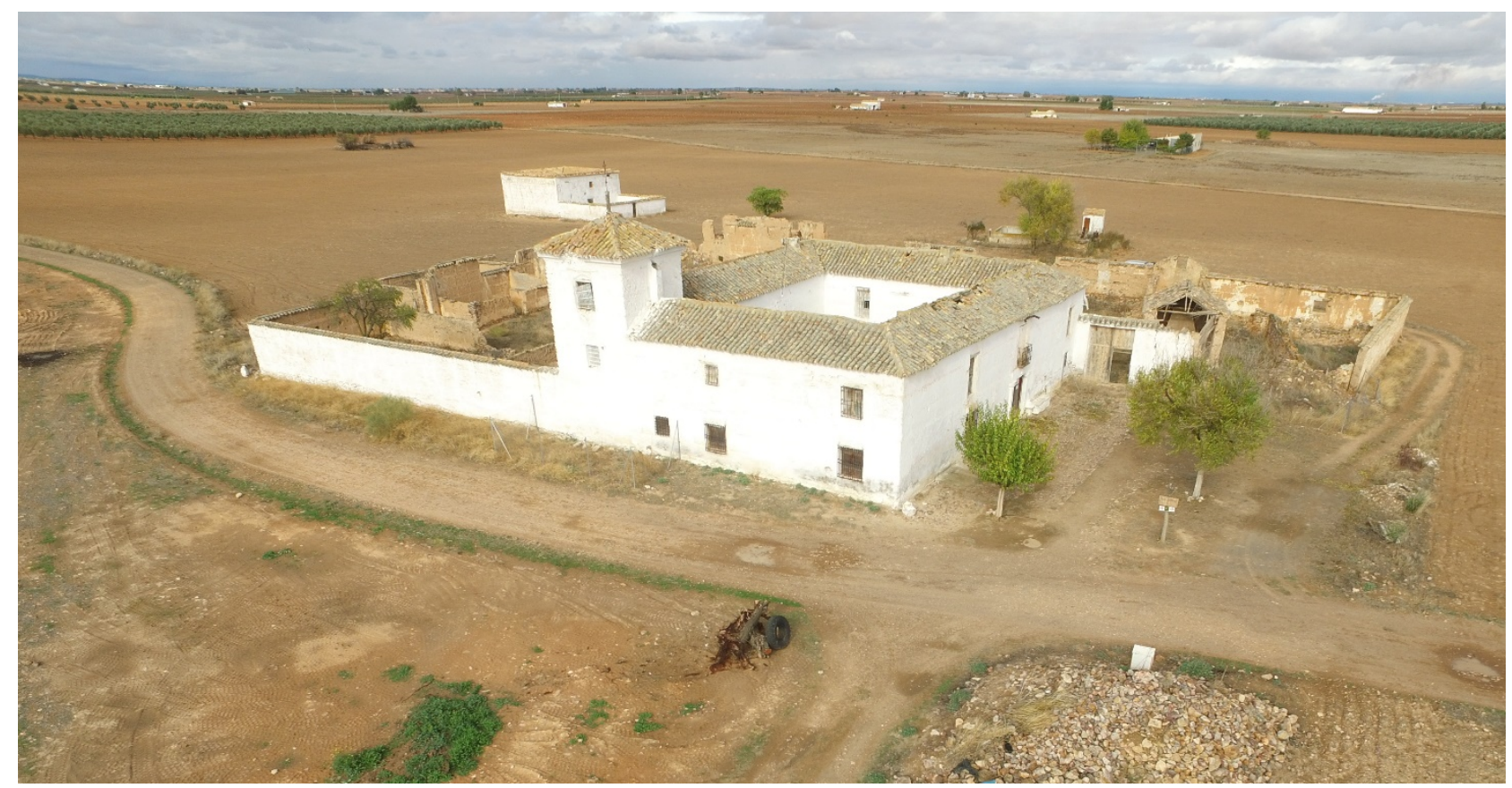

[Fotografia de Timoteo Ubeda-Portugues]. (Venta de Borondo. 2019). Archivo fotográfico personal.

Fig. 3 Fotografía aérea de Venta de Borondo

Este proyecto, que tiene por título Venta de Borondo «la venta que pudo ver Cervantes», analiza la situación real del edificio explicando las dificultades que presenta su restauración y puesta en valor. Conscientes de esas dificultades planteamos un proyecto sostenible y sostenido en el tiempo que se ejecutaría en seis fases, siendo la primera fase una intervención de urgencia sobre el edificio principal, restaurándose el torreón y las cubiertas. La fase final sería la puesta en valor; sin embargo, esta evolucionaría adaptándose a los espacios restaurados y abiertos al público. La propia restauración en sí es una gran oportunidad para impulsar la filosofía del "abierto por obras" y fomentar la formación de nuevos expertos en el estudio y restauración de la arquitectura tradicional ${ }^{1}$.

A la vez se han realizado diversas acciones culturales, desde conferencias a visitas guiadas, con el objetivo de divulgar y poner en valor el monumento. Sin embargo, la actividad más importante y de mayor éxito es la Jornada Cervantina que se celebra anualmente en conmemoración del día del Libro (Fig. 4) y que consiste en una ruta por el entorno de la venta, por lo alto de Sierra Pelada, pasando por un antiguo puente y un olivar con ejemplares de casi mil años. La ruta termina en la Venta de Borondo donde se procede a la lectura de varios capítulos de El Quijote.

La AC Venta de Borondo ha tenido claro desde un primer momento que la venta debía tener un mantenimiento anual. En una primera fase se procedió a la limpieza de estancias, patios y exteriores, retirando la vegetación que ocultaba el empedrado del patio; y en una segunda fase se realizó un primer encalado de las paredes, protegiéndolas de la erosión producida por las inclemencias del tiempo. Sin embargo, la posibilidad del derrumbe del torreón continuaba estando presente, y no había respuesta por parte de la administración competente.

La oportunidad llegó cuando la Fundación Soliss organizó el concurso Semilla Soliss 2017, donde la ciudadanía castellano-manchega podía elegir mediante votación on-line cuáles serían los cinco proyectos sociales, culturales o deportivos, que recibirían de esta fundación 6.000 euros. El proyecto de intervención de urgencia sobre el torreón de la Venta de Borondo resultó ser el segundo más votado, consiguiéndose la primera financiación privada para comenzar a soñar con salvar la venta. El hashtag \#SalvemosBorondo cada vez estaba más cerca de cumplirse.

\footnotetext{
${ }^{1}$ Esta propuesta se presentó en Legatum 2.0. II Congreso Internacional de Musealización y Puesta en Valor del Patrimonio Cultural celebrado los días 16, 17, 18 y 19 de octubre de 2018 en Daimiel, Ciudad Real (Cejudo, 2018).
} 


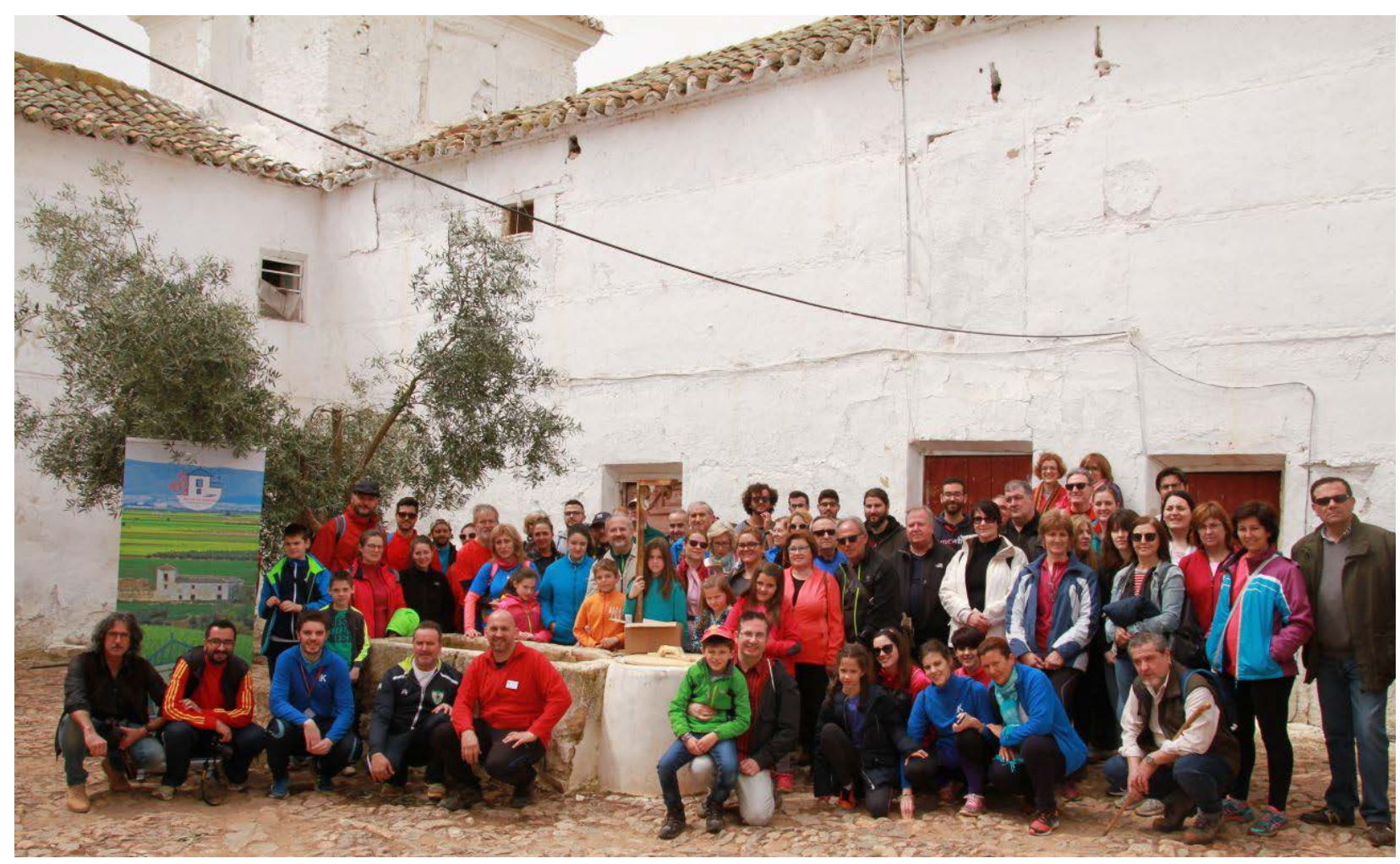

[Fotografía de Asociación Cultural Venta de Borondo y Patrimonio Manchego]. (Venta de Borondo. 2018). Archivo fotográfico de la asociación

Fig. 4 II Jornada Cervantina

La repercusión de la venta se incrementó en prensa y redes sociales, llegando a la asociación nuevas propuestas y formas de colaboración. Una de las más importantes fue la iniciativa de la familia Aranda Palmero, la mujer y las hijas del estadístico José Aranda Aznar decidieron reeditar su novela "La Venta de Borondo"2, donde el escritor nos contaba la historia de una joven arquitecta que por azar terminaba involucrada en la restauración del edificio. Los beneficios de la venta irían íntegramente a financiar un proyecto con el que había soñado su padre veinte años atrás.

La financiación se completaría con la obtención de una subvención de 13.267,65 euros procedente de las ayudas que destina cada año Parques Nacionales de Castilla-La Mancha a sus áreas de influencia socioeconómica, en concreto se optó a las ayudas que se dirigen a la restauración de edificios de arquitectura tradicional. Estaba todo preparado para comenzar a conseguir los permisos necesarios para comenzar la intervención de urgencia.

Cabe destacar la implicación de la empresa de soluciones digitales para el patrimonio 7reasons Media, quién se ofreció a realizar la digitalización de la Venta de Borondo antes del inicio de las obras. Se realizó un modelo virtual mediante fotogrametría con la inestimable colaboración de la operadora de vuelo A vista de RPAZ, permitiendo la documentación exhaustiva de la venta (Fig. 5).

A finales de 2018 se ejecutaría la restauración del torreón (Figura 6), un hito para la historia de la asociación. La restauración ha permitido evitar el derrumbe de la parte más simbólica de este conjunto arquitectónico. Una actuación de gran urgencia que ha sido asumida por la propia asociación con el objetivo de demostrar la viabilidad de una puesta en valor en fases. La obra tuvo lugar entre los meses de noviembre y diciembre bajo la dirección técnica del arquitecto Teodoro Sánchez-Migallón y la dirección arqueológica de Miguel Torres Más, Honorio Javier Álvarez García y la restauradora M. ${ }^{a}$ Isabel Angulo Bujanda.

${ }^{2}$ Esta novela fue reeditada por la editorial Hoja del Monte en el año 2017. 


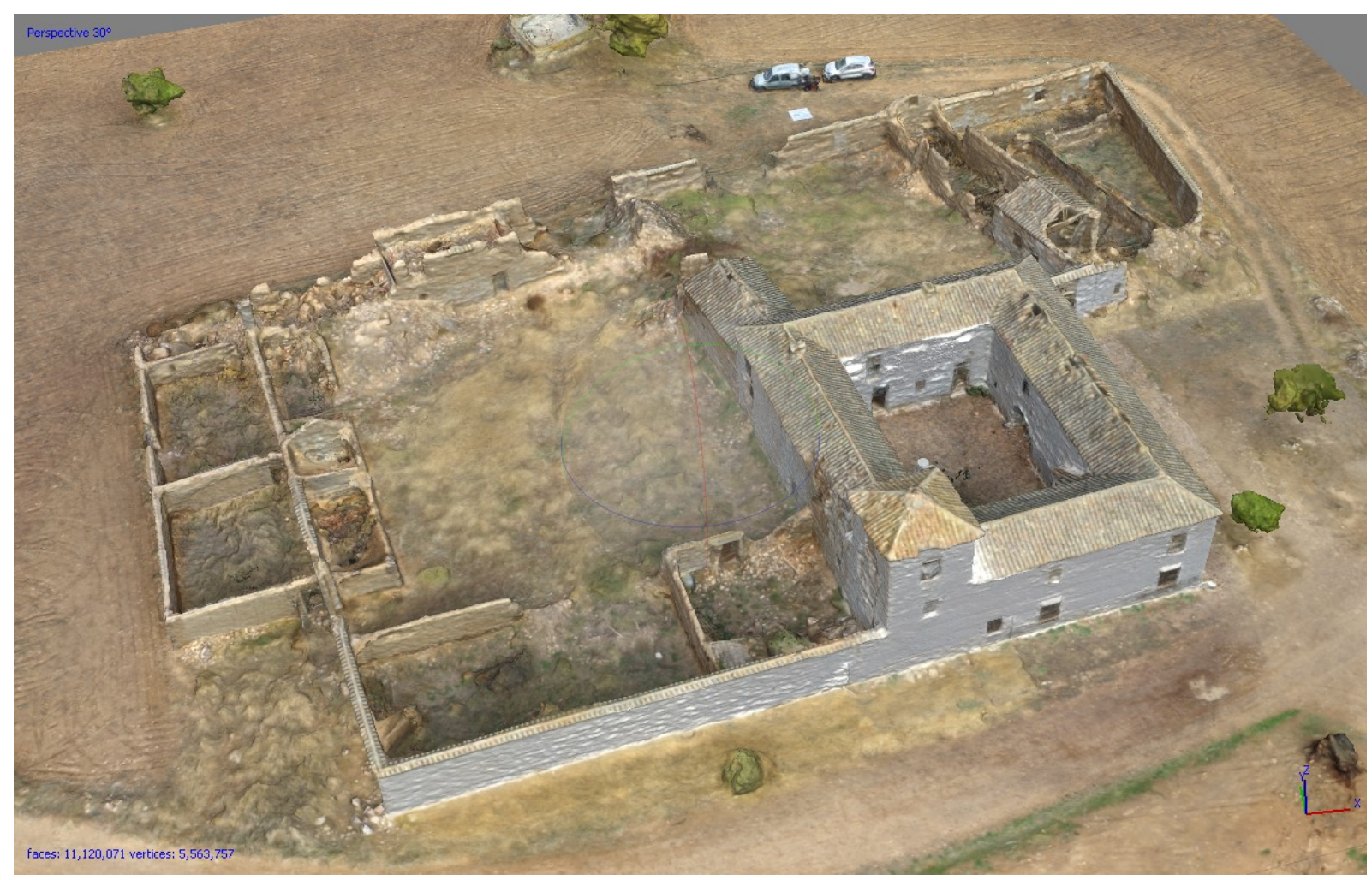

Fuente: 7reasons Media (2018)

Fig. 5 Modelo digitalizado Venta de Borondo

[Fotografía de Julio Orellana]. (Venta de Borondo. 2018). Archivo fotográfico personal Fig. 6 Trabajos de restauración del alzado oeste y el torreón 
Durante la intervención de urgencia se han estabilizado aquellos muros, cubiertas y aleros que afectaban a la estabilidad del torreón suroeste. Para su ejecución se ha seguido el principio de mínima intervención, reutilizándose materiales y el empleo de materiales tradicionales compatibles con los sistemas constructivos tradicionales siguiendo las directrices del Servicio de Patrimonio y Arqueología de la Junta de Comunidades de Castilla-La Mancha. Una acción posible gracias a implicación de números agentes que han aportado distintos recursos. Además del apoyo económico mencionado anteriormente, distintas empresas locales han apoyado la iniciativa como: J. García Carrión y Ferroal. Por su parte el Ayuntamiento de Daimiel se ha comprometido públicamente a aceptar las donaciones del monumento con el objetivo de continuar con las labores de restauración.

\section{Conclusiones}

La falta de concienciación social respecto a la arquitectura tradicional rural ha llevado a la pérdida de numerosos ejemplos arquitectónicos como: bombos, casillas de quintería, silos o casas-cueva. La Venta de Borondo podría ser un ejemplo más de "casa de campo" abandonada y víctima de la indiferencia de todos; sin embargo, gracias al interés mostrado por figuras como Miguel Fisac o José Aranda por proteger esta venta de llanura del siglo XVI posibilitó su catalogación como Bien de Interés Cultural. Esta protección no supuso su restauración en un primer momento, pero si el reconocimiento de su valor patrimonial y a la larga posibilitó la conformación de un movimiento social a favor de su defensa, restauración y puesta en valor.

La proliferación en los últimos años de asociaciones en defensa del patrimonio cultural nos habla de una sociedad preocupada por el legado arquitectónico de sus antepasados. La AC Venta de Borondo es un buen ejemplo de ello, marcando las líneas que pueden seguir otras asociaciones que deseen trabajar por el patrimonio cultural. La asociación continuará divulgando los valores de la venta (Figura 7) y de la arquitectura tradicional manchega, la necesidad de su defensa y protección, las posibilidades para la formación de profesionales en arquitectura, restauración o arqueología, y las oportunidades que ofrecen para el desarrollo económico en zonas afectadas por la despoblación.

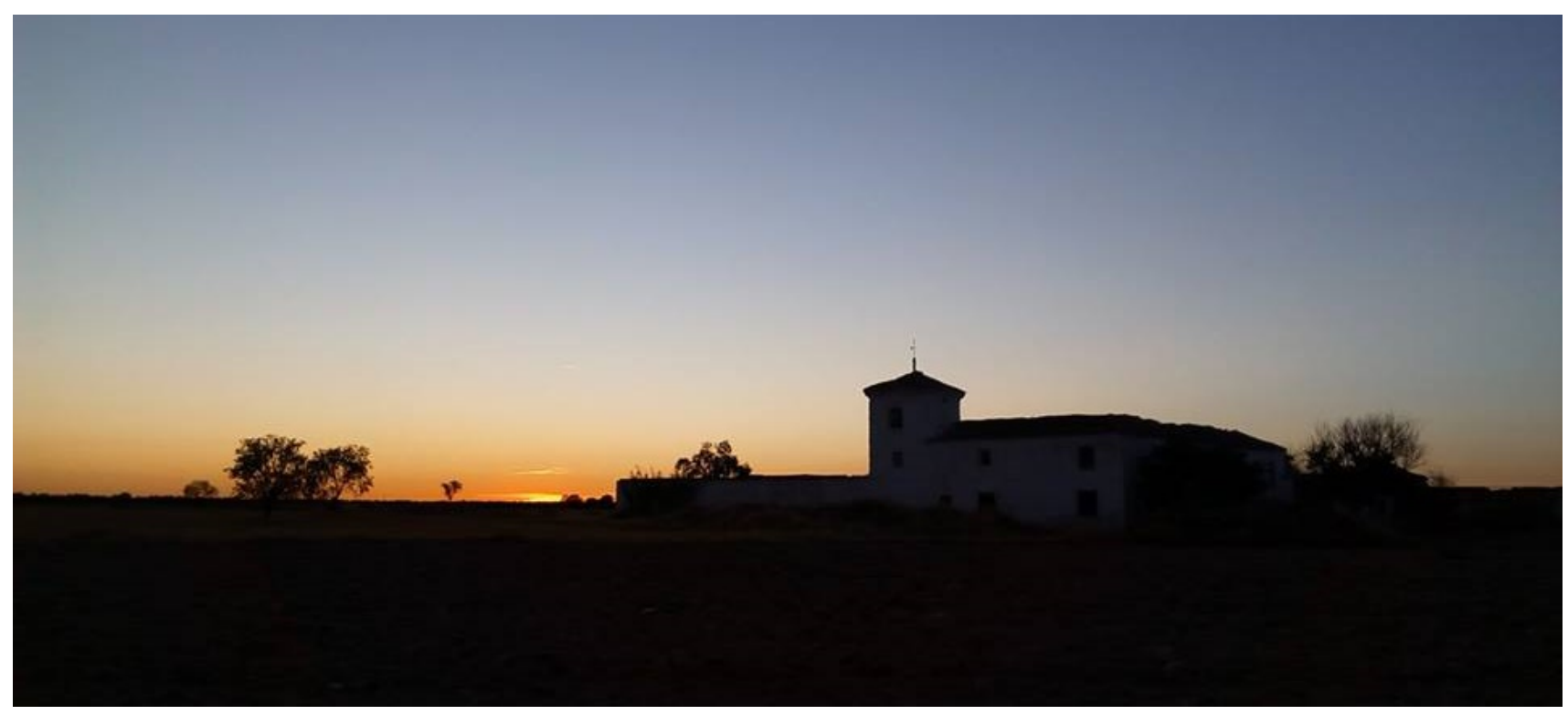

[Fotografia de David Cejudo]. (Venta de Borondo. 2018). Archivo fotográfico personal

Fig. 7 Atardecer en la Venta de Borondo

\section{Referencias}

Anónimo (1790). Carta escrita por Don Quixote de La Mancha a un pariente suyo en que se hace saber varias cosas necesarias para la perfecta inteligencia de la historia. Dala al público un paisano y apasionado de ambos. Madrid: Imprenta de Blas Román. 
Cejudo Loro, D. (2012). Proyecto de Intervención en la Venta de Borondo. Daimiel (Ciudad Real). Trabajo Final de Grado. Cuenca: Universidad de Castilla-La Mancha, Escuela Politécnica de Cuenca.

Cejudo Loro, D. (2013). Venta de Borondo. Origen y evolución hasta nuestros días. En II Jornadas de historia de Daimiel: 125 aniversario ciudad de Daimiel (pp. 71-84). Daimiel, Ciudad Real: Ayuntamiento de Daimiel.

Cejudo Loro, D., y Orellana López de la Franca, J. (2018, 19 de octubre). Patrimonio Cultural Ligado al Quijote. Alternativas de futuro para la Venta de Borondo. En J. Onrubia Pintado y V. M. López-Menchero Bendicho (Dir.), Legatum 2.0: II Congreso de Musealización y Puesta en Valor del Patrimonio Cultural. Comunicación llevada a cabo en el congreso Universidad de CastillaLa Mancha, Daimiel, Ciudad Real.

Dotor Municio, A. (1925). Por la España ignorada y pintoresca. Ruidera, el famoso lugar manchego de los lagos maravillosos. En Blanco y Negro. Recuperado de http://hemeroteca.abc.es/nav/Navigate.exe/hemeroteca/madrid/blanco.y.negro/1925/08/ 30/049.html

Junta de Comunidades de Castilla-La Mancha (2007). Resolución de 5 de marzo de 2007, de la Dirección General de Patrimonio y Museos, de la Consejería de Cultura, por la que se incoa expediente para declarar bien de interés cultural, con categoría de monumento, el inmueble correspondiente a la «Venta de Borondo», localizado en Daimiel (Ciudad Real). En Boletín Oficial del Estado, de 13 de abril de 2007, 89, 16.436-16.438. Recuperado de https://www.boe.es/boe/dias/2007/04/13/pdfs/A1643616438.pdf.

López, T. (1780). Mapa de una porción del Reyno de España que comprenden los parages por donde anduvo Don Quixote, escala: 20 leguas de una hora de camino, de las que entran 20 en un grado [= 7,9 cm] [ca. 1:1.402.200], [Madrid], Joaquín Ibarra. Recuperado de http://bdh-rd.bne.es/viewer.vm?id=0000193763 .

Campos y Fernández de Sevilla, F. J. (2009). Los pueblos de Ciudad Real en las Relaciones Topográficas de Felipe II. Ciudad Real: Diputación de Ciudad Real. Recuperado de http://publicaciones.dipucr.es/puebloscrealrelacionestopograficas.pdf . 